\title{
On the use of isoparametric elements for BEM modeling of arbitrarily shaped thin wires in electromagnetic compatibility applications
}

\author{
D. Poljak, V. Doric \& D. Cavka \\ University of Split, Croatia
}

\begin{abstract}
The paper deals with the frequency domain Galerkin-Bubnov scheme of the Indirect Boundary Element Method (GB-IBEM) for the solution of the Pocklington integral equations for arbitrary thin wire configurations. The presence of a lossy media is taken into account by using both the reflection coefficient approximation and the rigorous Sommerfeld integral formulation. Some illustrative computational examples related to power line communication systems (PLC) and grounding systems are presented.
\end{abstract}

Keywords: boundary elements, frequency domain modeling, Pocklington equation, thin wires of arbitrary shape.

\section{Introduction}

The most rigorous approach to analyze the electromagnetic field coupling to arbitrary configurations of overhead and buried wires, respectively, is related to the use of the antenna theory (the full wave approach) $[1,2]$. Such a formulation is usually based on a corresponding set of the Pocklington integral equations $[3,4]$. If one deals with the frequency domain formulation the related transient response is evaluated via the Inverse Fourier Transform (IFT). An important feature of the integral equation approach is the use of isoparametric elements for an efficient treatment of curved wires, which is not readily the case with a transmission line approximation [4]. On the other hand, the most serious drawback of the antenna theory is relatively long computational time required for the analysis of complex structures [1,2].

The excitation of aboveground and belowground wire configurations is of particular interest in electromagnetic compatibility (EMC). The knowledge of 
the induced current distribution is a prerequisite to understand the behaviour of related scattered fields, induced voltages and other parameters of the structure. The simplest procedure is to approximate the current distribution along the given wire structure. However, many applications require a more accurate approach related to the solution of a corresponding integral equation $[1,2]$.

The present paper overviews the analysis methods of electromagnetic field coupling to overhead and belowground wires in the frequency domain by means of the wire antenna model. Furthermore, the Galerkin-Bubnov scheme of the Indirect Boundary Element Method (GB-IBEM) for the solution of Pocklington equations is discussed. Some illustrative computational examples pertaining to overhead wires and grounding systems are given in the paper.

\section{Integral equation formulations for arbitrary wire structures}

This section outlines the basic principles in the analysis of field coupling to arbitrarily shaped overhead and buried wire configurations via the antenna theory approach. In both cases the formulation features the theory of images thus replacing either upper or lower half space respectively, as depicted in Fig 1.

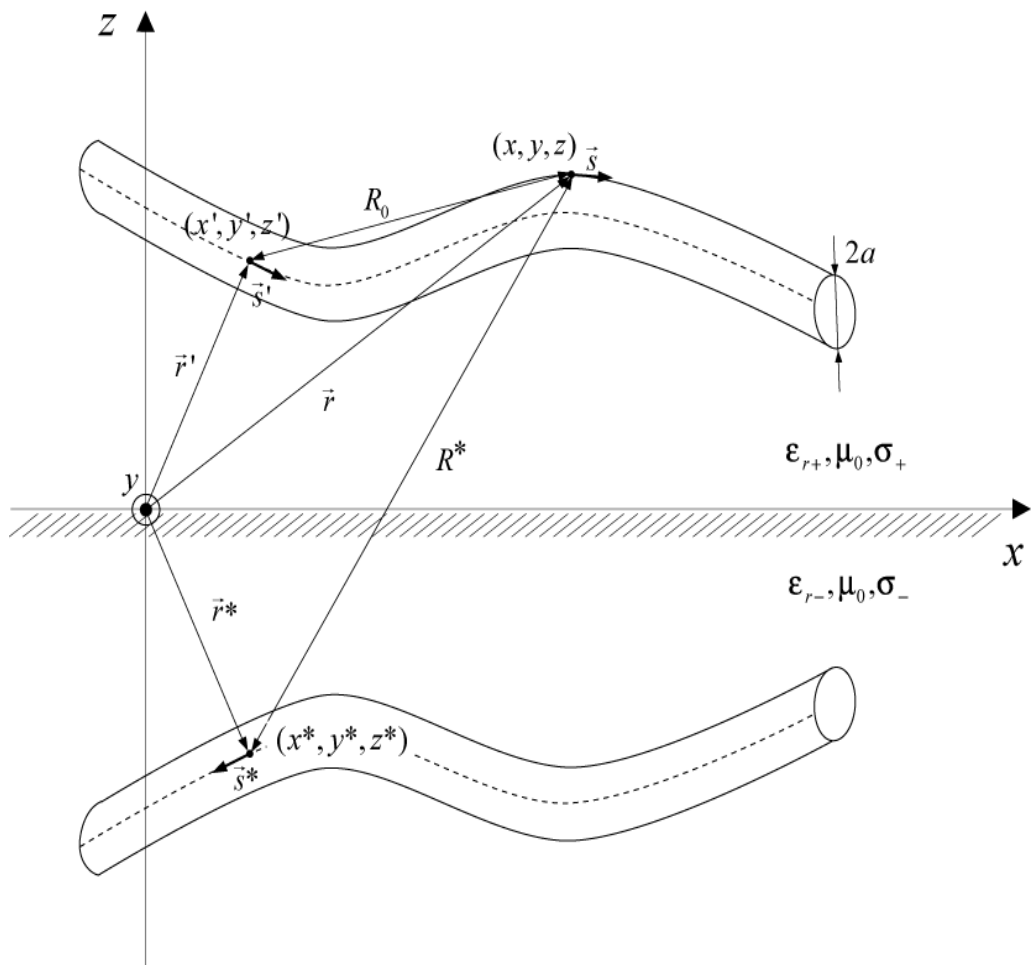

Figure 1: The wire of an arbitrary shape and its image. 


\subsection{Overhead wire configurations}

The currents $I\left(s_{n}^{\prime}\right)$ induced along multiple curved wires located above a lossy ground are governed by the set of corresponding Pocklington integral equations given by [5]:

$$
\begin{aligned}
& E_{s m}^{e x c}\left(s_{m}\right)=\frac{j}{4 \pi \omega \varepsilon_{0}} \sum_{n=1}^{N_{w}} \int_{0}^{L_{n}}\left\{\left[k^{2} \vec{e}_{s_{m}} \vec{e}_{s_{n}}-\frac{\partial^{2}}{\partial s_{m} \partial s_{n}^{\prime}}\right] g_{0 n}\left(s_{m}, s_{n}^{\prime}\right)+R_{T M}\left[k^{2} \vec{e}_{s_{m}} \vec{e}_{s_{n}}-\frac{\partial^{2}}{\partial s_{m} \partial s_{n}^{*}}\right] g_{i n}\left(s_{m}, s_{n}^{*}\right)+\right. \\
& \left.\left(R_{T E}-R_{T M}\right) \vec{e}_{s_{m}} \vec{e}_{p} \cdot\left[k^{2} \vec{e}_{p} \vec{e}_{s^{*}}-\frac{\partial^{2}}{\partial p \partial s^{*}}\right] g_{i}\left(s_{m}, s_{n}^{*}\right)\right\} I\left(s_{n}^{\prime}\right) d s^{\prime}+Z_{L m} I\left(s_{m}\right), \quad m=1,2, \ldots N_{w}
\end{aligned}
$$

where $N_{w}$ is the total number of wires, $I_{n}\left(s_{n}{ }^{\prime}\right)$ is the unknown current distribution along the $n$-th wire, $\vec{e}_{p}$ is the unit vector normal to the incident plane while $g_{0 m n}\left(x, x^{\prime}\right)$ and $g_{i m n}\left(s, s^{\prime}\right)$ are the Green functions of the form:

$$
g_{0 m n}\left(s_{m}, s_{n}^{\prime}\right)=\frac{e^{-j k R_{1 m n}}}{R_{1 m n}}, \quad g_{i m n}\left(s_{m}, s_{n}^{\prime}\right)=\frac{e^{-j k R_{2 m n}}}{R_{2 m n}}
$$

where and $R_{I m n}$ and $R_{2 m n}$ are distances from the source point and from the corresponding image, respectively to the observation point of interest.

The influence of a lossy half-space is taken into account via the Fresnel plane wave reflection coefficient (RC) for TM and TE polarization, respectively [5]:

$$
\begin{gathered}
R_{T M}^{\prime}=\frac{\underline{n} \cos \theta^{\prime}-\sqrt{\underline{n}-\sin ^{2} \theta^{\prime}}}{\underline{n} \cos \theta^{\prime}+\sqrt{\underline{n}-\sin ^{2} \theta^{\prime}}} \\
R_{T E}=\frac{\cos \theta^{\prime}-\sqrt{\underline{n}-\sin ^{2} \theta^{\prime}}}{\cos \theta^{\prime}+\sqrt{\underline{n}-\sin ^{2} \theta^{\prime}}}
\end{gathered}
$$

where $\theta^{\prime}$ is the angle of incidence and $\underline{n}$ is given by:

$$
\underline{n}=\frac{\varepsilon_{e f f}}{\varepsilon_{0}}, \quad \varepsilon_{e f f}=\varepsilon_{r} \varepsilon_{0}-j \frac{\sigma}{\omega}
$$

and $\varepsilon_{e f f}$ denotes the complex permittivity of the ground, while $\omega$ is the operating frequency.

Once the currents along the wire array are known, the radiated field components could be evaluated. The total electric field irradiated by configuration of arbitrarily shaped multiple wires is given by $[3,6]$ :

$$
\vec{E}=\sum_{n=1}^{N_{w}}\left[\vec{E}_{0 n}+R_{T M} \vec{E}_{i n}+\left(R_{T E}-R_{T M}\right)\left(\vec{E}_{i n} \cdot \vec{e}_{p}\right) \vec{e}_{p}\right]
$$

where:

$$
\vec{E}_{0 n}=\frac{1}{j 4 \pi \omega \varepsilon_{0}}\left[k_{1}^{2} \int_{0}^{L_{n}} \vec{e}_{s_{n}}{ }^{\prime} I\left(s_{n}{ }^{\prime}\right) g_{0 n}\left(\vec{r}, \vec{r}^{\prime}\right) d s_{n}{ }^{\prime}+\int_{0}^{L} \frac{\partial I\left(s_{n}{ }^{\prime}\right)}{\partial s_{n}{ }^{\prime}} \nabla g_{0 n}\left(\vec{r}, \vec{r}^{\prime}\right) d s_{n}{ }^{\prime}\right]
$$




$$
\vec{E}_{i n}=\frac{1}{j 4 \pi \omega \varepsilon_{0}}\left[k_{1}^{2} \int_{0}^{L_{n}} \vec{e}_{s_{n}{ }^{*}} I\left(s^{\prime}{ }_{n}\right) g_{\text {in }}\left(\vec{r}, \vec{r}^{*}\right) d w^{\prime}-\int_{0}^{L_{n}} \frac{\partial I\left(s^{*}\right)}{\partial s_{n}^{*}} \nabla g_{\text {in }}\left(\vec{r}, \vec{r}^{*}\right) d s^{\prime}\right]
$$

Note that index 0 and $i$ is related to the source and image wire, respectively. The total magnetic field can be determined from similar relations $[3,6]$.

\subsection{Buried wire configurations}

The currents induced along the complex grounding system can be obtained as a solution of the following set of the coupled Pocklington integro-differential equations for $N_{W}$ wires of arbitrary shape:

$$
E_{s m}^{e x c}\left(s_{m}\right)=-\frac{1}{j 4 \pi \omega \varepsilon_{e f f}} \sum_{n=1}^{N_{W}}\left[\begin{array}{l}
\int_{C_{n}{ }^{\prime}} I_{n}\left(s_{n}{ }^{\prime}\right) \cdot \vec{s}_{m} \cdot \vec{s}_{n}{ }^{\prime} \cdot\left[k_{1}^{2}+\nabla \nabla\right] g_{0 n}\left(s_{m}, s_{n}{ }^{\prime}\right) d s_{n}{ }^{\prime}+ \\
+\frac{k_{0}^{2}-k_{1}^{2}}{k_{0}^{2}+k_{1}{ }^{2}} \int_{C_{n}{ }^{\prime}} I_{n}\left(s_{n}{ }^{\prime}\right) \cdot \vec{s}_{m} \cdot \vec{s}_{n} * \cdot\left[k_{1}^{2}+\nabla \nabla\right] g_{i m m}\left(s_{m}, s_{n}{ }^{*}\right) d s_{n}{ }^{\prime}+ \\
+\int_{C_{n}{ }^{\prime}} I_{n}\left(s_{n}{ }^{\prime}\right) \cdot \vec{s}_{m} \cdot \vec{s}_{n} * \cdot G_{s}\left(s_{m}, s_{n}{ }^{\prime}\right) d s_{n}{ }^{\prime}
\end{array}\right], \quad m=1,2, \ldots, N_{W}
$$

where $k_{0}$ and $k_{l}$ are propagation constants of air and lossy ground, respectively:

$$
\begin{aligned}
& k_{0}^{2}=\omega^{2} \mu_{0} \varepsilon_{0} \\
& k_{1}^{2}=\omega^{2} \mu_{0} \varepsilon_{e f f}
\end{aligned}
$$

while $g_{0 \mathrm{~nm}}$ and $g_{\text {inm }}$ are the corresponding Green functions [4]. The kernel:

$$
\vec{G}_{s}\left(s, s^{\prime}\right)=\left(\vec{e}_{x} \cdot \vec{s}^{\prime}\right) \cdot\left(G_{\rho}^{H} \cdot \vec{e}_{\rho}+G_{\phi}^{H} \cdot \vec{e}_{\phi}+G_{z}^{H} \cdot \vec{e}_{z}\right)+\left(\vec{e}_{z} \cdot \vec{s}^{\prime}\right) \cdot\left(G_{\rho}^{V} \cdot \vec{e}_{\rho}+G_{z}^{V} \cdot \vec{e}_{z}\right)
$$

consists of the following terms [7]:

$$
\begin{gathered}
G_{\rho}^{V}=\frac{\partial^{2}}{\partial \rho \partial z} k_{0}^{2} V^{R} \\
G_{z}^{V}=\left(\frac{\partial^{2}}{\partial z^{2}}+k_{1}^{2}\right) k_{0}^{2} V^{R} \\
G_{\rho}^{H}=\cos \varphi\left(\frac{\partial^{2}}{\partial \rho^{2}} k_{1}^{2} V^{R}+k_{1}^{2} U^{R}\right) \\
G_{\phi}^{H}=-\sin \phi\left(\frac{1}{\rho} \frac{\partial}{\partial \rho} k_{1}^{2} V^{R}+k_{1}^{2} U^{R}\right) \\
G_{z}^{H}=-j 4 \pi \omega \varepsilon_{\text {efec }} \cos \phi G_{\rho}^{V}
\end{gathered}
$$

Where the Sommerfeld integral terms are:

$$
U^{R}=\int_{0}^{\infty} D_{1}(\lambda) e^{-\gamma_{1}\left|z+z^{\prime}\right|} J_{0}(\lambda \rho) \lambda d \lambda, D_{1}(\lambda)=\frac{2}{\gamma_{0}+\gamma_{1}}-\frac{2 k_{1}^{2}}{\gamma_{1}\left(k_{1}^{2}+k_{0}^{2}\right)}
$$




$$
V^{R}=\int_{0}^{\infty} D_{2}(\lambda) e^{-\gamma_{1}\left|z+z^{\prime}\right|} J_{0}(\lambda \rho) \lambda d \lambda, D_{2}(\lambda)=\frac{2}{k_{1}^{2} \gamma_{0}+k_{0}^{2} \gamma_{1}}-\frac{2}{\gamma_{1}\left(k_{1}^{2}+k_{0}^{2}\right)}
$$

where

$$
\gamma_{0}=\sqrt{\lambda^{2}-k_{0}^{2}} ; \quad \gamma_{1}=\sqrt{\lambda^{2}-k_{1}^{2}}
$$

Integrals (18) and (19) are computed numerically [4].

\section{Numerical solution of Pocklington type equations}

The set of Pocklington integro-differential equations (1) and (9) is handled via the (GB-IBEM) featuring isoparametric elements.

The unknown current $I_{n}^{e}(\zeta)$ along the $n$-th wire segment is expressed by the sum of a finite number of linearly independent basis functions $f_{n i}$, with unknown complex coefficients $I_{n i}$ :

$$
I_{n}^{e}(\zeta)=\sum_{i=1}^{n} I_{n i} f_{n i}(\zeta)=\{f\}_{n}^{T}\{I\}_{n}
$$

where $n$ is the number of local nodes per the element.

\subsection{BEM procedures for overhead wires}

Applying the weighted residual approach and performing the Galerkin-Bubnov procedure the set of coupled integro-differential equations (1) is transformed into the following matrix equation $[3,6]$ :

$$
\sum_{n=1}^{N_{w}} \sum_{\mathrm{i}=1}^{N_{n}}[Z]_{\mathrm{ji}}^{\mathrm{e}}\{I\}_{\mathrm{i}}^{\mathrm{e}}=\{\mathrm{V}\}_{\mathrm{j}}^{\mathrm{e}}, \mathrm{m}=1,2, \ldots, N_{w} ; \quad \mathrm{j}=1,2, \ldots, N_{m}
$$

where $N_{\mathrm{w}}$ is the total number of wires, while $N_{m}$ and $N_{\mathrm{n}}$ denotes the number of elements on the $m$-th and the $n$-th wire, respectively.

The mutual impedance matrix is given by $[3,6]$ :

$$
\begin{aligned}
& {[Z]_{-j}^{e}=-\int_{-1}^{1} \int_{-1}^{1}\{D\}_{j}\left\{D^{\prime \prime}\right\}_{i}^{T} g_{0 n m}\left(s_{n}, s_{m}^{\prime}\right) \frac{d s_{m}^{\prime}}{d \xi^{\prime}} d \xi^{\prime} \frac{d s_{n}}{d \xi} d \xi+k_{1}^{2} \vec{e}_{s_{n}} \vec{s}_{s_{m}} \int_{-1}^{1} \int_{-1}^{1}\{f\}_{j}\left\{f^{\prime}\right\}_{i}^{T} g_{0 n m}\left(s_{n}, s_{m}^{\prime}\right) \frac{d s_{m}^{\prime}}{d \xi^{\prime}} d \xi^{\prime} \frac{d s_{n}}{d \xi} d \xi-} \\
& -R_{T M} \int_{-1}^{1} \int_{-1}^{1}\{D\}_{j}\left\{D^{\prime}\right\}_{i}^{T} g_{i n m}\left(s_{n}, s_{m}^{*}\right) \frac{d s_{m}^{\prime}}{d \xi^{\prime}} d \xi^{\prime} \frac{d s_{n}}{d \xi} d \xi+R_{T M} k_{1}^{2} \vec{e}_{s_{n}} \vec{e}_{s_{m}^{*}}^{1} \int_{-1}^{1} \int_{-1}^{1}\{f\}_{j}\left\{f^{\prime}\right\}_{i}^{T} g_{i n m}\left(s_{n}, s_{m}^{*}\right) \frac{d s_{m}^{\prime}}{d \xi^{\prime}} d \xi^{\prime} \frac{d s_{n}}{d \xi} d \xi \\
& +\frac{j}{4 \pi \omega \varepsilon_{0}} \int_{-1}^{1} Z_{T}^{\prime}\{f\}_{j}\left\{f^{\prime}\right\}_{j}^{T} \frac{d s_{n}}{d \xi} d \xi
\end{aligned}
$$

while the voltage vector is $[3,6]$ :

$$
\{V\}_{j}^{n}=-j 4 \pi \omega \varepsilon_{0} \int_{-1}^{1} E_{s_{n}}^{e x c}\left(s_{n}\right)\{f\}_{j} \frac{d s_{n}}{d \xi} d \xi_{n}
$$


Matrices $\{f\}$ and $\left\{f^{\prime}\right\}$ contain the shape functions while $\{D\}$ and $\left\{D^{\prime}\right\}$ contain their derivatives. Once the current distribution is obtained, the radiated field can be obtained applying the similar BEM formalism $[3,6]$.

Thus, the total field is given by:

$$
\vec{E}=\sum_{k=1}^{N}\left[\vec{E}_{S k}^{e}+R_{T M} \vec{E}_{I k}^{e}+\left(R_{T E}-R_{T M}\right)\left(\vec{E}_{I k}^{e} \cdot \vec{e}_{p}\right) \vec{e}_{p}\right]
$$

where:

$$
\begin{aligned}
& \vec{E}_{S k}^{e}=\frac{1}{j 4 \pi \omega \varepsilon_{0}} \sum_{i=1}^{n}\left[k^{2} \int_{-1}^{1} \vec{e}_{k s^{\prime}} I_{i k}^{e} f_{i}(\xi) g_{0 k}\left(\vec{r}, \vec{r}^{\prime}\right) \frac{d s_{k}{ }^{\prime}}{d \xi} d \xi+\int_{-1}^{1} I_{i k}^{e} \frac{\partial f_{i}(\xi)}{\partial \xi} \nabla g_{0 k}\left(\vec{r}, \vec{r}^{\prime}\right) \frac{d s_{k^{\prime}}{ }^{\prime}}{d \xi} d \xi\right] \\
& \vec{E}_{I}^{e}=\frac{1}{j 4 \pi \omega \varepsilon_{0}} \sum_{i=1}^{n}\left[k^{2} \int_{-1}^{1} \vec{e}_{k s^{*}} I_{i k}^{e} f_{i}(\xi) g_{i k}\left(\vec{r}, \vec{r}^{*}\right) \frac{d s_{k}{ }^{\prime}}{d \xi} d \xi-\int_{-1}^{1} I_{i k}^{e} \frac{\partial f_{i}(\xi)}{\partial \xi^{\prime}} \nabla g_{i k}\left(\vec{r}, \vec{r}^{*}\right) \frac{d s_{k}{ }^{\prime}}{d \xi} d \xi\right]
\end{aligned}
$$

Numerical procedures for the computation of radiated magnetic field are presented in $[3,6]$.

\subsection{BEM procedure for Pocklington equation for buried wires}

Applying the weighted residual approach featuring the Galekin-Bubnov procedure the set of Pocklington equations (9) is transformed into a system of algebraic equations:

$$
\sum_{n=1}^{N_{w}} \sum_{i=1}^{N_{n}}[Z]_{j i}^{e}\left\{I_{n}\right\}_{\mathrm{i}}=0, \quad \mathrm{~m}=1,2, \ldots, N_{w} ; \quad \mathrm{j}=1,2, \ldots, N_{m}
$$

where the mutual impedance matrix is of the form:

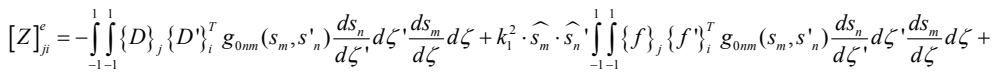

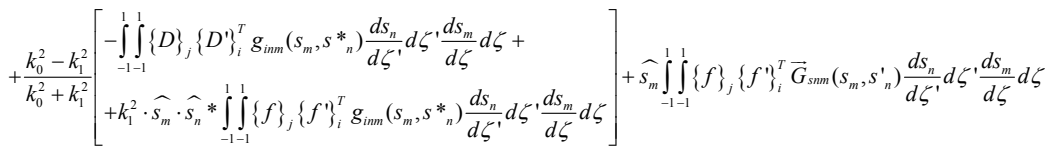

Note that the current source excitation $I_{\mathrm{g}}$ is taken into account as a forced boundary condition at a certain node $i$ of the grounding system [4, 7]:

$$
I_{i}=I_{g}
$$

The treatment of wire junctions is related to the Kirchhoff's current law forcing the continuity of currents and charges at the junction [4]. The input impedance of the grounding system is given by:

$$
Z_{\text {in }}=-\frac{1}{I_{g}} \int_{\infty}^{r} \vec{E} d \vec{s}
$$

where the line integral represents the driving point voltage. 
The desired frequency response of the grounding system is obtained by multiplying the input impedance spectrum with the excitation spectrum. The transient response is calculated by means of the IFFT.

\section{Computational examples}

The computational examples are related to a simple power line communications system and a realistic grounding system for wind turbines.

\subsection{PLC system analysis}

PLC technology provides users with communication means via the existing distributed power line network or electrical installations. Electromagnetic interference (EMI) is one of the principal drawbacks of this technology as overhead power lines radiate at PLC frequencies (1 MHz to $30 \mathrm{MHz}$ ) [6].

Fig 2 shows the geometry of a simple PLC system. The conductors are modelled as thin wires excited by the voltage generator $V_{g}$ at one end, and terminated by the load impedance $Z_{L}$ at the other end.

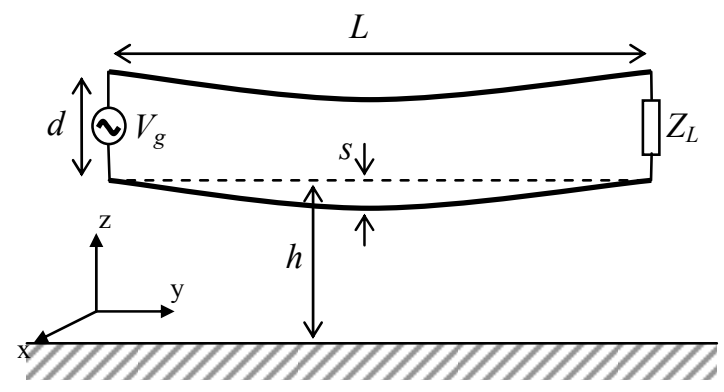

Figure 2: $\quad$ A simple PLC system.

The wire radius is a $=6.35 \mathrm{~mm}$, and the distance between poles is $L=200 \mathrm{~m}$. The conductors are suspended on the poles at height $h=10 \mathrm{~m}$ and $11 \mathrm{~m}$, respectively, while the maximal conductor sag is $s=2 \mathrm{~m}$. The maximal conductor sag is $s=2 \mathrm{~m}$. The lossy ground parameters are $\varepsilon_{r}=13$ and $\sigma=0.005 \mathrm{~S} / \mathrm{m}$.

The impressed power is $2.5 \mu \mathrm{W}$ (minimum power required for the PLC system operation) and operating frequency is $14 \mathrm{MHz}$. The value of the terminating load $Z_{L}$ is $500 \Omega$.

Fig 3 shows the radiated electric field, at the distance of $30 \mathrm{~m}$ from the wires and $10 \mathrm{~m}$ above a lossy ground. 


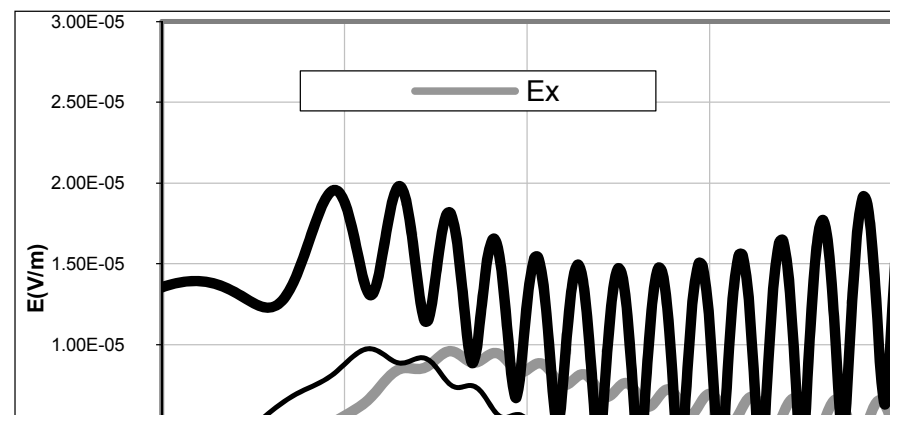

Figure 3: Radiated electric field from the PLC system.

\subsection{Transient analysis of wind turbine grounding system}

Installation of entire lightning protection system for wind turbines has become of particular importance $[8,9]$. Namely, wind turbines are quite often struck by lightning due to their special shape, complex construction and the fact that they are usually placed in isolated locations, mainly at higher altitudes. Therefore, low-impedance grounding system is a major prerequisite for a satisfactory protection of wind turbines from lightning strikes.

Fig 4 shows an arbitrary wind turbine grounding system subjected to a lighting strike. Note that the influence of WT itself (tower, blades etc.) is neglected.

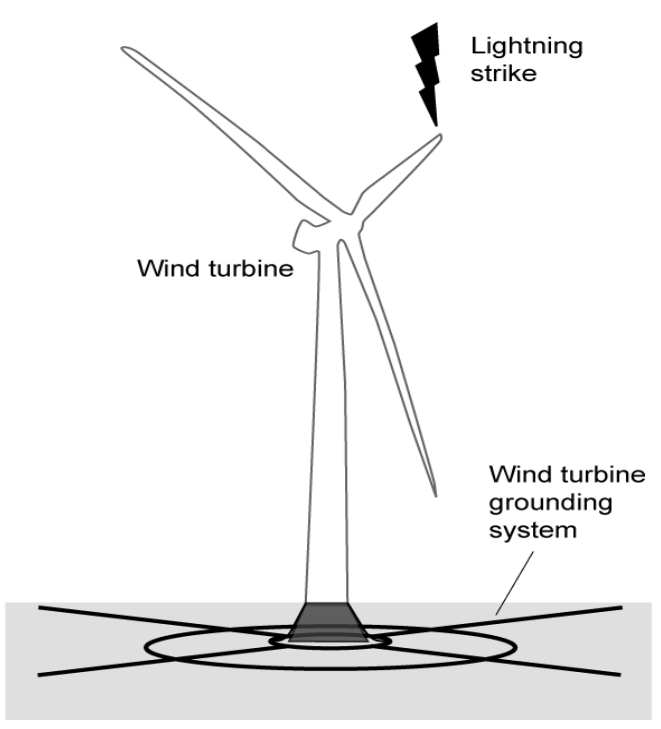

Figure 4: Wind turbine subjected to a lightning strike. 
Fig 5 shows a typical configuration of grounding systems for wind turbines. The grounding system consists of a square of galvanized steel flanges ( $\mathrm{Fe} / \mathrm{Zn}$ $30 \times 3.5 \mathrm{~mm}$ - gray line in fig 2) at the $2 \mathrm{~m}$ depth, two copper ring wires $(\mathrm{Cu} 70$ $\mathrm{mm}^{2}$ - black line in fig 2) at different levels (smaller one of $3.25 \mathrm{~m}$ radius at $5 \mathrm{~cm}$ depth and the larger with $6.8 \mathrm{~m}$ radius buried at $55 \mathrm{~cm}$ depth) and additional four copper wires. All parts of the grounding system are connected by aluminothermy welding.

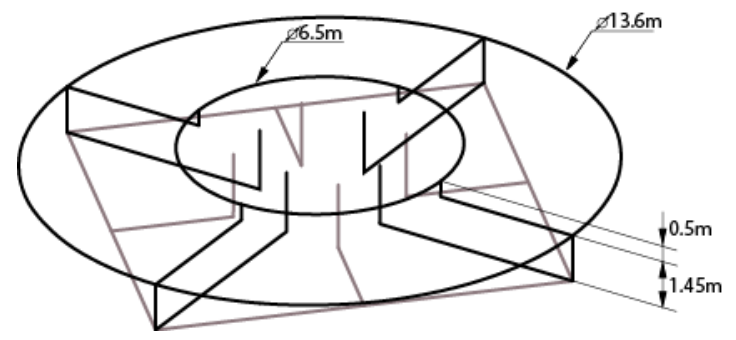

Figure 5: Typical wind turbine grounding system arrangement.

The grounding system is placed in a homogenous soil of relatively high specific resistance of $\rho=1200 \Omega / \mathrm{m}$ and the relative dielectric constant $\varepsilon_{r}=9$.

The lightning current is expressed by the double exponential function:

$$
i(t)=I_{0}\left(e^{-\alpha t}-e^{-\beta t}\right)
$$

with: $\mathrm{I}_{0}=1.1043 \mathrm{~A}, \alpha=0.07924 \cdot 10^{6} \mathrm{~s}^{-1}, \beta=0.07924 \cdot 10^{6} \mathrm{~s}^{-1}$.

Fig 6 shows the transient response of the grounding system. Dashed line represents a ten times higher input current waveform for the comparison purpose.

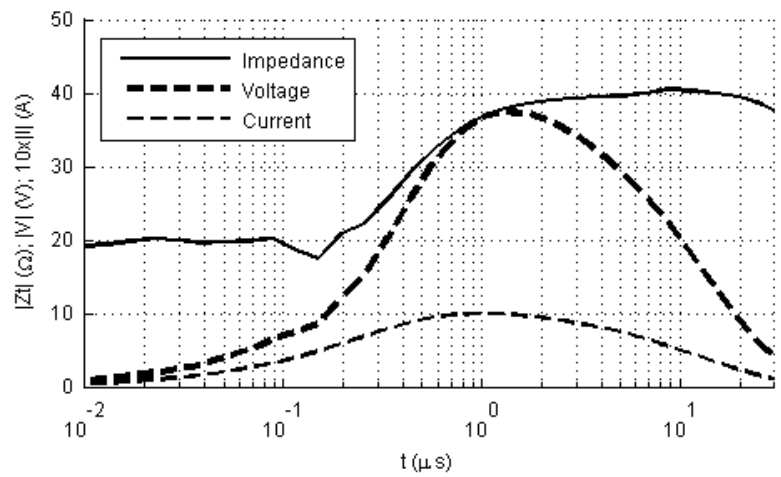

Figure 6: Transient behavior of the grounding system. 
It can be seen that the maximal value of voltage is about $37 \mathrm{~V}$ and it is reached slightly after current peak value. Transient impedance continuously increases from zero to maximal value of $40 \Omega$.

\section{Closure}

The paper deals with the frequency domain Galerkin-Bubnov scheme of the Indirect Boundary Element Method (GB-IBEM) for the solution of Pocklington integral equations for an efficient treatment of arbitrarily shaped wires featuring the use of isoparametric elements.

The presence of a dissipative half-space is taken into account by using both reflection coefficient approximation for overhead wires and rigorous Sommerfeld integral formulation for buried conductors, respectively.

Some illustrative numerical results pertaining to PLC systems and grounding systems are given in the paper.

\section{References}

[1] F. M. Tesche, M. Ianoz, and T. Karlsson, EMC Analysis methods and computational models. New York: Wiley Interscience, 1997.

[2] D.Poljak, EMC Advanced Modelling in Computational electromagnetic Compatibility, John Wiley and Sons, New York 2007.

[3] D.Poljak, V. Doric, M. Milisic, M.Birkic, Modeling of Array of Logperiodic Dipole Antennas Array for Air Traffic Applications, ICECom 2010, Dubrovnik, Croatia, Sept. 2010.

[4] Cavka D, Harrat B, Poljak D, Nekhoul B, Kerroum K, Drissi KEK. Wire Antenna Versus Modified Transmission Line Approach To The Transient Analysis Of Grounding Grid, Engineering Analysis with Boundary Elements, Vol. 3, 2011, pp 1101-1108.

[5] Miller EK, Poggio AJ, Burke GJ, Selden ES. Analysis of wire antennas in the presence of a conducting half-space, part II: the horizontal antenna in free space. Can J Phys 1972; 50:2614-27.

[6] V.Doric, D.Poljak, EMC Analysis of the PLC System Based on the Antenna Theory, ICECom 2010, Dubrovnik, Croatia, Sept. 2010.

[7] Grcev L, Dawalibi F. An Electromagnetic Model for Transients in Grounding Systems, IEEE Trans. on Power Delivery, Vol. 5, No. 4, pp 1773-1781, November 1990.

[8] IEC International Standard, "Wind Turbine Generation System - 24: Lightning Protection", IEC 61400-24 (2010). International Electrotechnical Commission, Geneva.

[9] IEA: "Recommended practices for wind turbine testing and evaluation, 9. Lightning Protection for Wind Turbine Installations.”, Ed. 1997. 